\title{
Secrecy Reconsidered: Parents of Children with Autism Spectrum Disorder (ASD) React to a Children's Book on the Subject of Autism
}

\author{
Boganim Orli, Baratz Lea \\ Achva College, Arugot, Israel \\ Email:orlyboga@gmail.com, lbaratz@netvision.net.il
}

How to cite this paper: Orli, B. and Lea, B. (2019) Secrecy Reconsidered: Parents of Children with Autism Spectrum Disorder (ASD) React to a Children's Book on the Subject of Autism. Open Access Library Journal, 6: e5456.

https://doi.org/10.4236/oalib.1105456

Received: May 8, 2019

Accepted: June 8, 2019

Published: June 11, 2019

Copyright $\odot 2019$ by author(s) and Open Access Library Inc.

This work is licensed under the Creative Commons Attribution International License (CC BY 4.0).

http://creativecommons.org/licenses/by/4.0/

\begin{abstract}
This study presents a qualitative narrative analysis of interviews conducted with 15 parents who have a child with autism spectrum disorder (ASD). This study describes the perceptions of parents of children with regarding the secret that is present in their lives, after reading the children's book. The theme of secrecy emerged indirectly and it exposed their experience as parents of a child with ASD. The book served as an instrument, on the one hand, while on the other hand, it constituted a medium that reflected the parents' experience relevant to ASD.
\end{abstract}

\section{Subject Areas}

Education

\section{Keywords}

Autism Spectrum Disorder, Parents of Children, Secret

\section{Theoretical Background}

A diagnosis of autism spectrum disorder (ASD) is a watershed event in the life of any family [1]. This "life-long adventure" requires numerous emotional resources; it evokes tumultuous emotions and internal contradictions, and often the family has difficulty finding a way to express its hidden secret [2]. Children's literature can serve as a major socialization resource for coping with and accepting a child with special needs [3]. A book can provide a helpful reflection that affords readers an in-depth view with clearer understanding of their emotions; it is a tool that mediates some of the sensitive issues and emotional processes that they may be undergoing. Through the medium of the story, par- 
ents can examine the reality described in the book as well as their own reality [4] in an indirect and nonthreatening manner [5].

The children's book I have a friend who is different, by Edna Mishori, deals openly with autism: it provides an opportunity to learn about a child who is different and about the difficulties experienced by this child and by those who surround the child. The story's protagonist is a boy named Doron, who has been diagnosed with ASD; he is different and yet he seeks to be in the company of other children. Enveloped in his own world, he is approached by another boy, Gadi, who wants to befriend him, despite the fact that Doron is not responding to his invitation. The story is narrated through the voice of Doron's mother, who wishes to expand his circle of friend. Through the story, she illuminates the unique world of children with autism as well as the complicated life voyage of the child's parents. The birth of a child with special needs shatters parents' expectations and hopes [6]. Parents of children with special needs undergo a difficult and painful process of shifting their attitudes and expectations. They are forced to accept the idea that their previous hopes and plans for the future are bound to change. Their coping process is characterized by fluctuations [1]. Although they start off with high hopes, they must relinquish these and instead accept the disability as an inseparable part of their lives. The impact and the length of the coping process differ from one person to another. In addition, parents tend to experience feelings of guilt and embarrassment, as well as contradictory feelings of love and joy on the one hand, and anger and disappointment, on the other hand. The broad range of difficulties that ASD entails also creates a great deal of stress and burdens the routine functioning of the child, which in turn can inhibit the development of various abilities [7]. Children with ASD who feel that their parents accept and understand them can feel secure in the company of their parents and will grow to rely on them increasingly, for emotional support to overcome moments of crisis, and for assistance in communicating their needs and difficulties, and as a medium for their own self-expression. The availability of such support can help reduce the intensity of characteristic impairments, thus enabling these children to strengthen their academic and functional capacities [8].

\subsection{Literature as a Cultural Agent}

Children's literature is perceived as a tool for molding the child's personal and intellectual character. It is characterized as contributing to three clearly defined aspects of development: the child's cognitive, mental, and linguistic skills; the development of social, ethical, and ideological mores, in accordance with the social norms of the child's surroundings; and the child's development as a potential reader who forms a sense of literary preference in preparation for entering the adult world [9]. Children's books present a variety of social situations taken from a child's world. The characters enact a variety of situations, which evoke emotions, desires, aspirations, and beliefs. Reading a children's book can develop 
the child's understanding of and ability to cope with complex social situations, and thus also an understanding of limitations [10].

The children's story in all its varieties is a window onto the human soul [10]. It serves as a therapeutic instrument in all cultures and is considered a powerful tool both in terms of its impact and its significance. The use of children's literature provides a way to reach the depths of one's soul and thus provide healing [11]. When we read, we do so in the hope of discovering something about who we are and where we stand in relation to others. Children's stories provide access to a person's hidden resources; they can stimulate the subconscious and open a path to creativity, in the pursuit of new answers to essential questions about our lives [12].

It is important to mediate the children's story to the young reader, so as to influence the child's array of future needs in terms of skill acquisition, thus increasing the child's motivation and ability to learn in the future. In the course of this mediation, one helps the child identify parts of the text that can provide guidance regarding moral values, while at the same time avoiding statements that might send a negative message [13]. Furthermore, providing such mediation teaches the child how to streamline the learning processes through self-mediation, and helps the child internalize various learning mechanisms and develop cognitive functions that are weak [14]. Children who experience proper mediation internalize the interaction and use it in later stages in a variety of contexts; they are able to actively initiate such interactions and regulate them on their own [15].

Furthermore, parents can benefit from the process of story mediation, as it adds to their personal experiences. The experience of mediated learning is considered a factor that explains the ability to undergo cognitive shifts and is defined as the person's tendency to learn from new experiences and opportunities and to change one's own cognitive structures [16]. Such changes to the cognitive structure are not automatic, but rather depend to a great extent on appropriately administered and suitable mediation. Delivering this mediation depends not only on parents' characteristics, but also on the strengths and cognitive weaknesses of the child, the child's motivations, emotional needs, behavioral tendencies, the type of stimuli involved, and the given situation [15].

The current study examined the manner in which the mediation of the children's story mentioned above affected the parents' reading of the story. Among the many insights derived from the interviews conducted with parents of children with ASD after having read the story, we discovered-hidden between the lines, so to speak-the theme of secrecy.

\subsection{The Essence of Secrecy}

Research on the topic of family secrets has been conducted-for the most part-from within the fields of social work, psychology, and family studies, and these studies typically analyzed the source of the secret and the family's func- 
tioning. However, every element of the social discourse on the topic of secrecy is interpretive [17]. There is no one overarching theory or model that describes how individuals make the decision to disclose or withhold a secret. Generally, researchers examine separate factors individuals take into consideration when deciding to disclose, such as privacy management, costs and benefits associated with disclosure, and power [18].

The word secret is derived from the Latin word secretus, which means to separate or to place aside, a root which is also present in the word secretaire, i.e., the place where one keeps objects that are private. Secretiveness plays an important role in the development of experience and in the formulation of the self, and "the core of the soul." is described as a secret. This is the hidden and private component that contains the uniqueness of the self: it is unknown and should remain unrevealed. A secret is defined as something hidden, an event, an experience, or information known only to oneself. The owner of the secret keeps it concealed from others, but not from the self [19]. The secret defines the boundary that separates "those who know" from "those who do not know," [20] and the secret is what unites those in the know [21]. The keeping of family secrets can be destructive, despite the fact that the main function of a secret is to protect [22].

In referring to a secret, it is important to distinguish between an active process of keeping a secret, that is, the decision to remain silent about a specific topic or event, and "silence for survival", whereby the need to survive creates the need for secrecy. In the former case, the silence helps in the construction of the private self, defining one's individualism and thus differentiating between the private and the public self. In the latter case, the silence might be motivated by humiliation, guilt, or social stigma, which creates the need for secrecy [23].

A secret can manifest in various ways [24]. There is the secret that one owns and of which one is aware [2], that is, when the possessor of the secret wishes to prevent its content from reaching others. The essence of this secret is others' lack of knowledge, and it can become a source of torment if it comes under attack or is revealed. The repressed secret refers to something threatening that is kept hidden in the individual's subconscious. The threat can be anything that remains harmless as long as it is concealed. The individual feels simultaneously drawn to and repelled by the secret and the need to cope with it. Occasionally, the difficulty of coping with these conflicting feelings leads one to refuse out-of-hand ownership of or any link to the secret [25]. A different approach refers to secrecy as "a potential space" [26], where one can exist within the self in complete privacy. A deviation in a person's developmental process is liable to convert this secret space into a characteristic of secretiveness in one's personality. Persons in trauma can either hide internally and express only the symptoms of trauma, or they can disappear entirely as they withdraw into their secret space. Finally, the secret concealed in the margin of consciousness is placed aside so that the owner of the secret remains unaware of it, even as he or she makes an effort to guard it 
as such. At some level, the individual is aware of the existence of the secret, yet one hides it from oneself for fear of feeling internal discomfort and unease [25]. People keep secrets from reaching a conscious level when they are apt to discover a discrepancy or dissonance between the moral code according to which they were educated and their actions or thoughts; hence, concealing these thoughts from surfacing to a conscious level is a way to avoid the anxiety that their revelation would provoke.

\subsection{A Family Secrets}

As Tolstoy noted in the opening line of the novel Anna Karenina, "All happy families are alike; each unhappy family is unhappy in its own way." Borrowing from this notion, we might say that all families with a child with ASD face similar issues, but the way that each cope with the issue of secrecy is different. Every family forms its own core of unwritten rules and regulations and family members are taught to abide by these from a young age. These rules of communication formulate and reflect major aspects of the family's role [27]. Each family type should be related to different attitudes about what secrets are appropriate to disclose to family members [18]. A family secret is accompanied by feelings of shame and is related to what a person thinks about him or herself: "Do I live up to my own ideals?" It is also related to the way one thinks others perceive one: "Will I be accepted or rejected?" In some cases, the secret is kept guarded by the nuclear family over a long period of time, in an attempt to protect children from stress and discomfort and to allow them to develop in a protected sphere [2]. The parent's secret develops in three stages [2]. In the first stage, the parent encounters a difficult reality that constructs his or her potential space and causes a great deal of discomfort. In the second stage, this discomfort evokes conflicting feelings, which are accompanied by a strong sense of guilt, shame, and alienation. The parent is involved in an internal struggle in an effort to keep things secret. In the third stage, the parent becomes disassociated from his or her own feelings, to the point that the feelings themselves become a hidden secret. This is a secret that the parent rejects or severs from his or her conscious self. Given that these conflicting emotions are never expressed directly, the parent is likely to develop certain behavioral symptoms, such as overprotecting the child, becoming overly worried and adopting passive aggressive behaviors towards the child, developing somatic responses, and directing anger towards the medical team or towards oneself. Legitimizing the true feelings of guilt and shame that the parent experiences is an important step, recognizing emotions that are normal in a situation that is a departure from the social norm. The parent experiences a process that fluctuates between acceptance and rejection, hope and hopelessness, secrecy and openness, guilt and forgiveness, burden and relief, loneliness and emotional expression, inaction and action, fear and courage, loss and renewal, as well as the absence of meaning and a revealed significance [28].

Following the reading of a children's book that deals with the subject of aut- 
ism, we studied the emotions the experience evoked in parents of children with ASD. Given that these parents are dealing with a reality that was forced upon them, we were able to examine their external and internal worlds and consider the ways in which the experience of reading a book on the subject of autism led them to open up about the topic.

\section{Method}

\subsection{The Central Problem and the Goal of the Study}

The goal of the study was to examine the emotions evoked in parents of children with ASD following their exposure to a children's book on the subject of autism, and to determine the ways in which the book reflects the parents' perceptions regarding the secrecy surrounding their child's condition.

\subsection{Methodology}

This is a narrative-based, qualitative study, which sought to understand, interpret, and generalize the studied phenomenon [29]. This is particularly suitable in cases where one tries to understand a phenomenon and reveal factors and motives that were not considered so important at the outset, and which in retrospect are likely to be revealed as central from the standpoint of the participants in the research and of the researchers [30].

\subsection{The Study Population}

The study population consisted of 15 parents of children with ASD: one father and 14 mothers, who reside in the central region of the country of study, and come from an average socioeconomic background. Their ages ranged from 35 to 50 years of age. The parents were recruited through the social platform of Facebook. As they belong to a closed Facebook group intended solely for parents of children with ASD, the researcher requested the group manager's permission to join the group. After being accepted, the researcher uploaded a post which explained that the author was seeking to interview parents of children with ASD for research purposes. Many parents responded to the post and expressed their willingness to participate; some even explained that they hoped their participation would contribute to a deeper understanding of the child's disability, as a way of helping themselves and others in their situation. All participants responded voluntarily and received an explanation regarding the ethical constraints that would be upheld in the course of the study.

\section{The Research Instruments}

The children's story titled I have a friend who is different, by Edna Mishori, was sent to the parents prior to conducting interviews. During the interviews, parents were asked to describe how they felt about what they had read in the book and about their own reality. They were asked to consider the extent to which the book reflected their own lives and whether it helped them in any way to cope 
with the related difficulties. As the interview progressed and a feeling of trust was forged between the interviewer and the interviewee, additional and complementary questions were asked. The complementary questions were intended to reaffirm points mentioned at an earlier stage of the interview, or additional questions were posed to address aspects that had not been sufficiently developed or which had remained unclear [31]. During the interviews, an effort was made to carefully record and code the collected data [29].

In the first stage of the interview, the parents were asked to respond with descriptions. Questions were open-ended and invited the participants to tell their own story in relation to what they had read, without addressing any particular angle [31]. Thus, parents were asked to describe their feelings towards the character in the story. At a later stage, the questions asked for more specific descriptions of examples related to interviewees' own lives, vis-à-vis the content of the book, as well as by free association. Throughout the course of the interviews, an effort was made to maintain a balance between emotional involvement and empathy, on the one hand, and emotional distance and critical thinking, on the other hand.

\subsection{Data Analysis}

The transcribed interviews were read by two experts in narrative analysis. The first stage of analysis consisted of open-ended coding, whereby each interview was analyzed separately, paragraph by paragraph, in order to identify repeating themes. Then, similar thematic categories that were common to all of the interviews were named. The second stage consisted of axial coding, after the categories had been defined, the relationships between the categories were formulated until a single major theme emerged.

\subsection{Ethical Considerations}

Every participant signed an informed consent form, to ensure that decisions were made independently [32]. Participants' anonymity and privacy were carefully maintained; hence, pseudonyms are used in the current study and identifying characteristics and backgrounds of the parents and children have been omitted intentionally.

\section{Findings}

In the course of the interviews, parents referred to the reality as described in the book, while comparing it with their own lives and emphasizing the extent to which the secret affects their lives. This theme was not addressed explicitly, but rather implicitly and through hints and clues, such that it reflects parents' experience of their role as parents of children with ASD. Their responses manifested a range of emotions, from identifying with the content of the book, through an emotional reaction of sadness and anger, as well as rational responses that aimed to examine their own reality. Herein, only responses related to the theme of the 
secret are described.

\subsection{The Many Faces of the Secret-Parents' Reflections}

The parents related to the notion of a secret, although they did not necessarily use the word secret explicitly. Some used anxiety as a tag word; others mentioned the need to conceal their situation, whereas others spoke of being closeted.

Although, as mentioned, parents were ensured of anonymity throughout the study, of the 15 interviewees, three mothers repeated the request that their privacy and anonymity be maintained and further asked that their life story should not be divulged in the study.

No one in our family knows, nor do any of our friends; I don't want people hinting about this, about our family. You can't tell how people will interpret whatever I say, so in general, I prefer that no one knows. (Nurit)

"I don't want to make this public for all the world to know, because then, you know... [there's no telling] how the neighbors and friends might react" (Tamar).

In contrast, other participants expressed things differently. "I don't have a problem if you even want to use our real names; it doesn't worry us. Anyone who knows us knows that our child has ASD. We're happy to share information so that everyone knows!" (Hani)

You don't even have to tell us about anonymity; we don't keep secretseverything is open and known. We don't hide and we're not ashamed. On the contrary, if I can help someone else, I'd consider it an enormous privilege. (Ronit)

\subsection{Stages in the Evolution of the Secret}

In the course of the interviews, participants described the experience of caring for a child with ASD. Some of the choices regarding the child's care and treatment had to do with the issue of secrecy, or as some of the parents described it: "we live a closeted life". This symbolic definition expresses the way in which they perceive their reality, i.e., forced to constrain the potential breadth of their lives in a manner that causes discomfort and is accompanied by internal and external conflicts, as well as by a restricting sense of guilt, shame, fear, and secrecy. As one parent (Irit) said, "Because autism isn't visible, you can try and hide it, and many of the parents do." In other words, many parents cope with an internal conflict, and when this conflict becomes dangerously burdensome, there is the risk that the parent might disassociate from reality and harbor negative feelings as an internal secret.

I haven't exactly outed us yet, you know, like people do when they come out of the closet, so to speak. We haven't even told our families. My husband's parents have no idea and my own parents know there's something... Also at school and with his friends-none of the mothers know, and I told no 
one at work either, not even my boss. No one! (Nurit)

[My child has] an accompanying tutor, who is supposedly unnoticed, but really there's no such thing. When we met her at school at first, my husband repeated endlessly: "we really ask that you don't make yourself noticed." But when we walked out, I told him I thought it [his effort] was pathetic: "do you really think no one will notice that everywhere our child goes the tutor goes too? You think no one will ask? Aside from the kids, the parents will want to know: what gives us the right to have a tutor present at all times? It's the way of the world!" (Nurit)

\subsection{Why Do We Keep It a Secret?}

Parents hesitated in choosing a term with which they felt comfortable describing their child's condition, as they did not wish to label their child. They mentioned personal issues related to shame, concern, and discomfort; hence, they elected to use expressions such as "the child has a communication disorder", "communication difficulties, a deficiency". Occasionally no definition was used at all. The attempt to avoid a definition is an attempt to escape, to pretend the problem does not exist; however, reliance on buzzwords reflects a situation in which the parents use softened or refined language to describe a phenomenon that is difficult and painful for them, in order to avoid calling it by its proper name. Occasionally, this is related to the surrounding environment, factors in the family, or concerns that the child might not be accepted in his or her environment, either by professionals or by the parents of his or her peers.

I don't tell people what the problem is exactly; I don't define it by name, I won't say the word autism, because of the stigma surrounding it. There are lots of people who don't even know what autism is, and they confuse it with delayed development or other things. That's why I say communication problems, because I don't think people have a sufficient understanding of what autism is. Autistic is currently used in society for name calling. I don't know if people know exactly what it is and I don't want to create the wrong impression about my son. I also don't want to go into extensive explanations with every person I meet: "look, you should understand, there's a spectrum..." That's not where I'm at. Instead, I use this kind of shortcut, an expression that I am sure represents things as accurately as possible. (Michal)

Irit noted:

People ask, people are curious; no one needs to know the exact terminology, but at the end of the day, people know, because that's life. We try to help him. If people are uncomfortable and don't want their child to be friends with mine, that's okay with me.

A study conducted [17] deals with parents' attempts to conceal their child's mental disability. The family creates a distinction between the internal family 
narrative, and the public narrative; but in reality, there is really only one story. The function of the separate narratives is to protect the family's "public image", revealing the details of the family's story could harm individual family members, as well as the family's public image [33].

The secretiveness comes into play in the closest social circle and with the immediate family. Many parents choose to conceal this information from their other children, for fear that these siblings will be unnecessarily burdened by the information or that they will not be able to cope with the information, conceptually and emotionally. Nurit revealed, "My mother knows that my child has an accompanying tutor at school and that he sees a communication therapist; my parents even help take my son to the therapy sessions, but they do not know the precise definition!”

My eldest child doesn't know. She knows that her sister has communication problems, but she doesn't know it's called autism, because autism still has a terribly negative connotation in our society. I think this situation has to change, but until then, I'm not going to use the word autism. (Tamar)

In the second, more distant social sphere, the participating parents contend with the school environment, as well as with the parents of their child's classmates, and with the neighbors. Efrat described how she manages things at school.

You know, the environment is made up of spheres. My parents and friends know. But, I don't necessarily share this everywhere. For example, I don't tell people at school, because I don't think it would help to have kids calling him names or something like that. I just say that there are some difficulties. If a classmate's parent asks me about the accompanying tutor, my answer is "yes, he has some social and communication difficulties," but I don't want to give the definition. I know from my own experience how it affected me to hear the term autism. You imagine a total catastrophe. So I don't want to make things more difficult for him; on the contrary, I want to make things easier whenever and in any way I can. (Tali)

Also, some educational professionals prefer to keep the term a secret, as Hani described.

The kindergarten teacher didn't want us to tell. We thought this was a major mistake; we did want to tell, but she forbade us. She said that some of the parents would make our lives miserable. I think it was silly.

This may have been the kindergarten teacher's way to cope with the social environment, or perhaps this choice was personally or professionally motivated.

\subsection{The Price of Secrecy}

Hiding, covering up, and keeping secrets, as well as manipulating or softening certain facts, all require an enormous emotional investment [2]. Traces of the 
internal battle within the soul of the parent are expressed through mental and physiological symptoms, and occasionally they obscure the link between the symptom and the secret [17].

I find it difficult to come out and say, "this is my child,"-that is my own weakness, so I'm constantly covering up. I'm still in the stage of feeling ashamed; yet I never expected such a child and I still can't come to terms with the fact that this is what I've got. It means constantly coping. When I think of whether to take him with me, I have to consider every aspect: where I'm going, whether he'll have bursts of rage, the length of the trip, whether he'll be able to cope with the people around. I can't just take him with me anywhere. It's not like her [the character in the story] who takes the child with her. When we are in the playground, I'm constantly hovering around him and protecting him. I find it difficult, because I'm left with a secret. I have to cover up or justify. There's something easy about just saying "okay, he has autism" without trying to explain or conceal it. She [the character in the story] is courageous and she also believes that it would be good for the child to find a friend. (Dina)

Another aspect of concealing the situation is related to the perceived need to justify oneself. The word justification reflects the parents' desire for justice, to prove they are not responsible for what happened, on the one hand, while on the other hand, it is an attempt to explain why they do not want to be exposed and the price of keeping the secret.

None of us asked for this and each of us copes as best as he or she can... I see around me parents who have a hard time coming to terms with it and they conceal the issue of the autism... We didn't steal or do anything wrong, so there's nothing for anyone to judge... Nevertheless, coming out is a [complicated] process... (Shira)

Keeping a secret reflects the belief that this is a personal and private matter and that it is related to a sense of guilt, failure, or prejudice [34]. In contrast, some parents are interested in sharing their feelings with the environment, as part of a worldview that encourages openness and transparency for the sake of diminishing lack of knowledge.

I made sure that they [the siblings] would tell their classmates that we have a child with autism, in order to increase awareness. I didn't want to have any secrets... My daughter was very active in her youth group and she was good at "selling" the ASD. We hide nothing. (Avi)

$\mathrm{He}$ is completely aware. We've always spoken about autism as if it were another friend at home. We never hid it or kept it secret. Today they are adolescents and it is a "fact". They are aware of what is going on with them... From the start, I never hid the fact that [their sibling] has some kind of problem. [In the book] I loved the part where she [the mother] says 
he has autism, that he experiences difficulties, and the way she explained the difficulties and the positive things. (Galit)

The book gave me hope that maybe I too can manage to be brave and say the things out loud: that this is the child and this is how you need to behave with him. Maybe this will open the way for me to be less protective and less secretive. (Dina)

After the diagnosis I deliberated whether or not to come out about it. At the end, he [the husband] convinced me. To this day I am not completely at peace with the step we took... Nowadays, society is more understanding; everybody's coming out about everything: homosexuality, illnesses, disorders - it's not as secretive as it used to be. There was a time when people with disorders were kept indoors and never appeared in public. So yes, things are improving, but still, the word autism is a difficult one. At the same time, I have to have some answer to give people who come and ask what the child has. (Irit)

Irit's comment summarizes the ambivalence with which parents of children with ASD must cope, an ambivalence that stretches across a continuum ranging all the way from denial to acceptance, from secrecy to openness and exposure. This ambivalence may surface as part of the way the parents' interpret their emotions and their surroundings vis-à-vis the child's condition; it is part and parcel of the need to construct a new narrative about a reality that is forever changed and irreversible. Perhaps the deliberation is resolved with the realization that secrecy does not solve the problem [17].

\section{Discussion and Conclusions}

In the course of the interviews about the role of the book, without being asked directly, without intending to, and occasionally even without being aware, parents revealed the role of secrecy in the process of coping with the issue of autism. The current study examined the perceptions and emotions evoked in parents of children with ASD following a reading of the children's book I have a friend who is different, which deals with autism. The research question aimed to demonstrate the way in which the reading process reflected the readers' own world to them. Findings indicated that secrecy was a focal point through which the parents related the story to their own world. In addition to the roles attributed to literature as an agent of socialization, verbal enrichment, and moral principles [35], it has been shown that literature can serve as a symbolic mirror of the readers' lives, from which readers can draw support and encouragement [4].

The first stage of the research was to present the book to the parents; from the interviews, we gathered information about their feelings and the role the book played for them. The fictional situation is multilayered and the story is symbolic [5], which gives the book a mediating role. The emphasis is on the importance of human mediation, based on the tendency to learn from new experiences and opportunities, a learning that entails changing one's own cognitive structures. By 
means of the book, important emotional processes were highlighted, which served a preliminary role in determining the type of mediation and its effects [15]. Books are recognized as an important therapeutic instrument in all cultures, an instrument known for its effects and its significance [12]. Hence, a book can serve as a therapeutic instrument also in the discourse on autism, a context in which it has not yet figured prominently. The book served as a factor that humanized the interaction between the outside world and the parents' private inner worlds. Reading the book created for them a new need, namely, to collect information so as to understand the world thus, it turned them into powerful and independent consumers.

The parents were exposed to a variety of possible actions and reactions, which spelled out for them an option of flexibility, creativity, and the ability to understand and generalize to insights beyond what is told in the story. At this stage, parents expressed frustration, pain, and anger, on the one hand, while on the other hand, they were given the opportunity to rethink the reality of their lives. The parents became aware of social and ethical needs and expressed a desire to change themselves, in order to contend more effectively with their situation. In fact, at this stage, the parents began to raise significant questions about their own actions and behaviors.

The term secret or secrecy was not used explicitly, but hinted at through expressions that describe concealment, pretense, and "coming out of the closet." According to the professional literature, parents tend to deliberate over a period of weeks, months, or years about the terminology they ought to use and with which they can feel comfortable relating to their child's condition [36]. They deliberate whether to share the information with the child and with others, and if so, with which family members. The fact that the word autistic is often used for name calling [8] prevents parents from using it when communicating with the surrounding environment and even amongst themselves.

Through the book, the parents were able to discern certain processes, which they had often blocked from themselves and from those surrounding them. A parent who keeps a secret is heavily invested in the secret, which is referred to as a conscious secret [2]. The parent is forced to cope with the enormous emotional burden of this internalized secret, until he or she can do so no longer, and the truth or parts of it seep out. In fact, due to the particular features of ASD, at a certain point it becomes impossible to continue keeping it hidden. Another scenario is the repressed secret, in which case the person feels simultaneously drawn and repelled by the object (the secret) and the need to cope with it. Occasionally, the difficulty of coping with this internal conflict can lead the parent to reject the object entirely.

The coping demonstrated by the parents participating in this study can be perceived in terms of three major groups. In the first group are the two mothers who were completely secretive and felt threatened by the possibility that the secret might be revealed and hence the disability remained completely repressed. 
The second group consisted of parents who were well aware of the essence of the disability and spoke of it openly without any fear, threat or attempt to repress the information. The third group consisted of the majority of the parents, whose stories demonstrated their sense of dissonance, trapped as they are between secrecy and openness. The secret was deeply hidden and the experience of reading the book penetrated their consciousness and made them aware of the repression caused by secrecy. In fact, parents in this group were aware of the secret they harbor within and of their ability to cope with it when they come in contact with others. This finding suggests that the experience of reading the book served as a catalyst, which enabled them to talk about the secret that they harbor. The book shed light or brought to the surface that which they knew to be true but which they refused to admit; it teased out the secret that had been hidden and about which they had refused to speak either with themselves or with those in their environment.

In the case of this third group, the secret is considered "hidden from the eye but close to the consciousness." It forms around a nucleus of anxiety, which leaves the person distant and unconnected, so that he or she turns inward to seek solace [2]. This third group is inhabited by parents whose stories are not revealed on Internet sites and who remain unreachable. When we examine the three stages as described by [2], that lead parents to harbor a secret, it is clear that the participants in the current study underwent the first two stages, although in their case, the third stage was somewhat different than that described in the model.

In the first stage, the parents encounter a new and difficult reality, which constrains their potential living arena and creates a feeling of discomfort. This stage is similar to that of receiving the news, before the stages of denial and acceptance. In the second stage, parents' discomfort creates internal conflicts and an emotional burden. In the current study, the internal conflict revolves around the secret. In the third stage-in the current context-in distinction from the model in which parents are described as removed from their own feelings to the point that these are not recognized, the facts become known and it is at this stage that parents must cope with the situation. Moreover, the parent becomes active, both at home in their immediate community and in society at large. In fact these parents do not become disassociated, but rather engage in activity for the sake of the child, each in his and her own way, according to their individual understanding and using their own unique tools [37]. The use of the book, which was intended to emphasize an experiential aspect, became a mediating tool for the parents' emotional processes. The book reflected the parent's issues that they previously dared not speak of, either with each other or in society. However, in the book, someone had written it all down in words [24].

The main contribution of the current study is in demonstrating that the book served as an experimental instrument and is the source of support and encouragement. The goal was not to reveal the secret, but rather to deal with its exis- 
tence and consider how it could be handled. Addressing parents' needs in a professional and experimental (sp.) manner can bring not only relief to the parents, but also help them tailor the environment to the needs of the child, culminating in a beneficial relationship with the child and with the child's immediate surroundings.

The parents, each in their own way, presented a personal interpretation of the book and gave it new meaning, based on stimuli, context, prior knowledge, empowerment and the personal and the cultural world of one's background. Stories serve as a window to the human soul. Hence, for every one of the participants in this study, the story was able to mirror their lives and serve as a source of support, encouragement, and empowerment. The book helped the parents find ways to cope with whatever was amiss in their way of handling the child's disorder vis-à-vis the communal and social spheres. The book made the parents more aware not only of ways to treat the child, but also more aware of the way they treat themselves. When the issue of their reality was placed in the spotlight, they had no choice but to come face-to-face with the truth.

\section{Conclusions and Recommendations}

Books in general can serve as a source of stimulus and as a mediating tool for parents of children with any type of disability. It also serves to increase the awareness of educators and therapists who treat children with ASD of the enormous emotional burden which these parents bear. It is important that professionals understand the potential effects of using books and thus can provide professional support and information to parents. Such help may diminish the internal conflicts with which parents of children with ASD cope throughout the course of their lives and help establish a better balance. Although the parents in the current study are not representative of all parents of children with ASD, the content that emerged from the interviews is certainly characteristic of this group (which group?) of parents. Limitations of the study included the small sample size, which prevents findings from being generalizable to all families of children with ASD; nevertheless, findings of the current study shed light on the complex reality with which these parents cope. The issue of secrecy and parents' emotional burden is an important aspect that should be further studied in future research.

\section{Conflicts of Interest}

The authors declare no conflicts of interest regarding the publication of this paper.

\section{References}

[1] Mishori, E. (2014) Life Journey with ASD—The Life Stories of Parents. MOFET Institute, Tel Aviv. (In Hebrew)

[2] Arelle-Brodsky, H. (2012) Secrets I Haven't Even Told Myself. Conversations, 27, 
49-55. (In Hebrew)

[3] Sacerdoti, Y. (2013) I'm Not Exactly What You Wanted-On the Encounter between a Typical Child and a Child with Special Needs in Israeli Children's Literature. Dvarim Acherim, 6, 133-149. (In Hebrew)

[4] Lindsay, N. (2006) Bringing Home the World. School Library Journal, 52, 36-37.

[5] Carta-Schwartz, S. (2012) "The Power of Story"-Applying a Symbolic Story as an Instrument of Communication and Therapy. (In Hebrew) http://www.hebpsy.net/articles.asp?id=2864

[6] Mishori, E. (1992) Autism and Communication Disorders: Options for Education and Coping. ALUT: The National Association for Children with Autism, Tel Aviv. (In Hebrew)

[7] Dolev, S. (2014) Parents' Acceptance of a Child's Diagnosis of Autism. In: Levinger, S., Ed., Over the Rainbow. Innovations in Diagnosis and Therapy for Children with $A S D$, Ach, Kiryat-Bialik, 9-33. (In Hebrew)

[8] Campbell, J. (2006) Changing Children's Attitudes toward Autism: A Process of Persuasive Communication. Journal of Developmental and Physical Disabilities, 18, 251-272. https://doi.org/10.1007/s10882-006-9015-7

[9] Sacerdoti, Y. (2000) Together and Separately-The Child Addressee and the Adult Addressee in the Discourse of Children's Literature. Hakibbitz Ha'Meuchad, Tel Aviv. (In Hebrew)

[10] Kurtts, S.A. and Gavigan, K.W. (2008) Understanding (Dis)abilities through Children's Literature. Education Libraries, 31, 23-31. https://doi.org/10.26443/el.v31i3.259

[11] Zoran, R. (2000) The Third Voice: The Therapeutic Qualities of Literature and Their Application in Bibliotherapeutic Dialogue. Carmel, Jerusalem.

[12] Baratz, L. and Kass, E. (2007) Bibliotherapy as a Tool to Prevent Eating Disorder. Mofet Institute, Tel Aviv, 175 p. (In Hebrew)

[13] Baratz, L. and Kass, E. (2011) The Adult as Message Mediator in Children's Books Dealing with Eating Disorder. Journal of Reading, Writing and Literacy, 5, 1-11.

[14] Tzuriel, D. (2011) Revealing the Effects of Cognitive Education Programmers through Dynamic. Assessment in Education: Principles, Policy \& Practice, 18, 115-131. https://doi.org/10.1080/0969594X.2011.567110

[15] Tzuriel, D. (2013) Mediated Learning Experience and Cognitive Modifiability. Journal of Cognitive Education and Psychology, 12, 59-80. https://doi.org/10.1891/1945-8959.12.1.59

[16] Tzuriel, D. (2014) Mediated Learning Experience and Cognitive Modifiability. Transylvanians Journal of Psychology, 35, 15-49.

[17] Burghardt, M. (2014) Narratives of Separation: Institutions, Families, and the Construction of Difference. Unpublished Doctoral Dissertation, York University, Toronto.

[18] Bostwick, E.N. and Johnson, A.J. (2018) Family Secrets: The Roles of Family Communication Patterns and Conflict Styles between Parents and Young Adult Children. Communication Reports, 31, 91-102. https://doi.org/10.1080/08934215.2017.1380209

[19] Aharoni, C. (2005) A Hidden Secret-Between Listening and Therapeutic Containing. Conversations, 19, 159-167. (In Hebrew)

[20] Vangelisti, A. and Caughlin, J.P. (1997) Revealing Family Secrets: The Influence of Topic, Function, and Relationships. Journal of Social and Personal Relationships, 14, 679-705. https://doi.org/10.1177/0265407597145006 
[21] Cohen, Y. (1992) The Secret and Its Role in Development. Conversations, 7, 20-25. (In Hebrew)

[22] Karpel, M.A. (1980) Family Secrets: Implications for Research and Therapy. Family Process, 19, 295-306. https://doi.org/10.1111/j.1545-5300.1980.00295.x

[23] Kauffman, T. (2008) The Hidden Secret: Mental Illness in the Family. (In Hebrew) http://www.hebpsy.net

[24] Zoran, R. (2007) Silence in Therapy and in Literary Texts-The Literary Text's Encouraging Function in Bibliotherapeutic Dialogue. In: Efrat, M., Ed., Silences: The Role of Silence in Culture and in Interpersonal Interactions, Reisling, Tel Aviv, 123-136. (In Hebrew)

[25] Bollas, C. (1984) The Shadow of an Object: Psychoanalysis of the Known But Unimportant. Krause, M. Translator, Dvir, Tel Aviv. (In Hebrew)

[26] Khan, R. (1983) From Secretiveness to Shared Living. In: Hidden Selves: Between Theory and Practice in Psychoanalysis, the International Psycho-Analytical Library, No. 113, Random House, London, 88-96. https://doi.org/10.4324/9780429475511-5

[27] Bergman, Z. and Cohen, A. (1994) The Family-A Journey to Common Ground. Am Oved, Tel Aviv. (In Hebrew)

[28] Navon, S., Feigin, R. and Drori, M. (2001) Paving the Way for Families Coping with Disease and Disability—Therapeutic Models. Tel-Aviv University, Ramot. (In Hebrew)

[29] Gvaton, D. (2001) Grounded Theory: The Meaning of the Data Analysis and Theory Construction in Qualitative Research. In: Tzabar Ben-Yehoshua, N., Ed., Studies of Raditions and Movements in Qualitative Research, Dvir, Tel Aviv, 195-221. (In Hebrew)

[30] Sabar Ben-Yehoshua, N. (2016) Traditions and Genres in Qualitative Research. Philosophies, Strategiesand Advanced Tools. Mofet Institution, Tel Aviv. (In Hebrew)

[31] Shkedi, A. (2011) The Meaning behind the Words. Methodologies in Qualitative Research-From Theory to Practice. Tel Aviv University, Ramot. (In Hebrew)

[32] Dushenik, L. and Tzabar Ben-Yehoshua, N. (2001) The Ethics of Qualitative Research. In: Tzabar Ben-Yehoshua, N., Ed., Studies of Traditions and Movements in Qualitative Research, Dvir, Tel Aviv, 343-368. (In Hebrew)

[33] Vangelisti, A. (1994) Family Secrets: Forms, Functions and Correlates. Journal of Social and Personal Relationships, 11, 113-135. https://doi.org/10.1177/0265407594111007

[34] Steffánsdóttir, G. and Traustadóttir, R. (2015) Life Histories as Counter-Narratives against Dominant and Negative Stereotypes about People with Intellectual Disabilities. Disability \& Society, 30, 368-380. https://doi.org/10.1080/09687599.2015.1024827

[35] Lintner, T. (2011) Using “Exceptional” Children's Literature to Promote Character Education in Elementary Social Studies Classrooms. The Social Studies, 102, 200-203. https://doi.org/10.1080/00377996.2010.550955

[36] Duek-Huri, S. (2014) Self-Discovery and Self-Advocacy: A Process of Therapeutic, Family-Based, and System-Wide Dialogue. In: Levinger, S., Ed., Over the Rainbow. Innovations in Iagnosis and Therapy for Children with ASD, Ach, Kiryat-Bialik, 235-270. (In Hebrew)

[37] Mishori, E. (2013) The Life Journey of Parents of Children with ASD. In: Avisar, G. and Reiter, S., Eds., Shilouvim: From Theory to Practice, AHVA Publishers, Haifa. (In Hebrew) 